\section{9th-Century Geometry}

C arl Friedrich Gauss (1777-1855) lived in Göttingen $7^{\text {and }}$ worked in many areas, ranging from complex numbers to astronomy and electricity. He gave the first satisfactory proof that every polynomial equation has a complex root, and in number theory he initiated the study of congruences and proved the law of quadratic reciprocity. He also determined which regular polygons could be constructed with ruler and compasses: his regular 17-gon is shown on the East German stamp.

Gauss was also interested in the foundations of geometry. Euclid's Elements commences with five "postulates." Four are straightforward, but the fifth is different in style. It

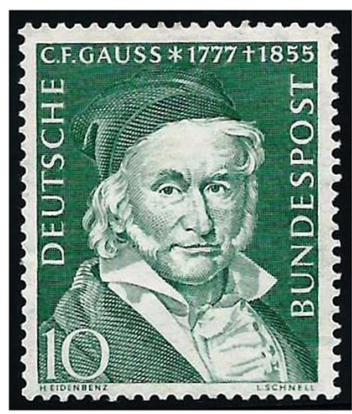

Gauss

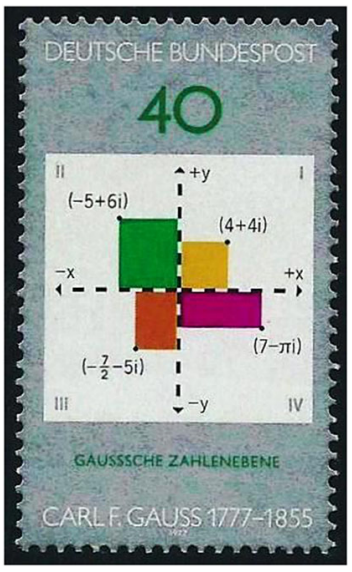

Gaussian plane

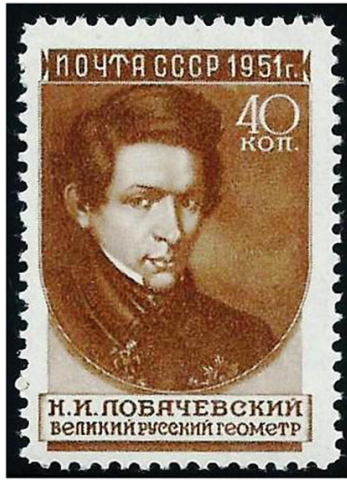

Lobachevskii is equivalent to the parallel postulate: given any line $l$ and any point $p$ not lying on $l$, there is a unique line through $p$ that is parallel to $l$. For more than 2000 years, mathematicians tried to deduce this result from the first four postulates, but they were unable to do so.

This is because there are "non-Euclidean geometries" satisfying the first four postulates but not the fifth. Such geometries have infinitely many lines through $p$ that are parallel to $l$, and they were discovered independently around 1830 by Nikolai Lobachevskii (1792-1856) in Russia and János Bolyai (1802-1860) in Hungary. When Bolyai's father outlined János's work to his friend Gauss, the latter dismissed it as something he had discovered previously but never published; the Bolyais never forgave Gauss for this. Neither Bolyai nor Lobachevskii gained widespread recognition for his efforts until after his death.

Another interesting geometrical object is the Möbius band, which has only one side and one boundary edge. First discovered by Johann Benedict Listing (1808-1882), it is named after the German mathematician and astronomer August Möbius (1790-1868), who described it in 1858.

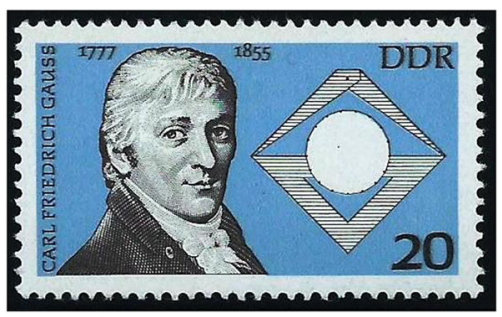

Gauss's I7-gon

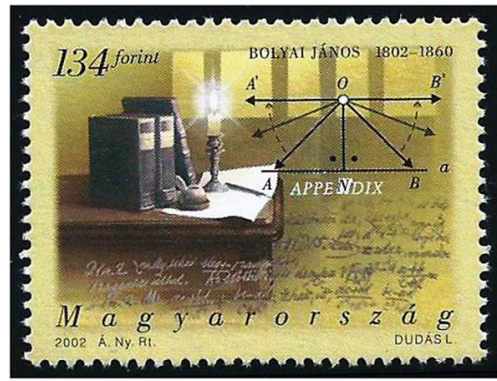

Bolyai's geometry

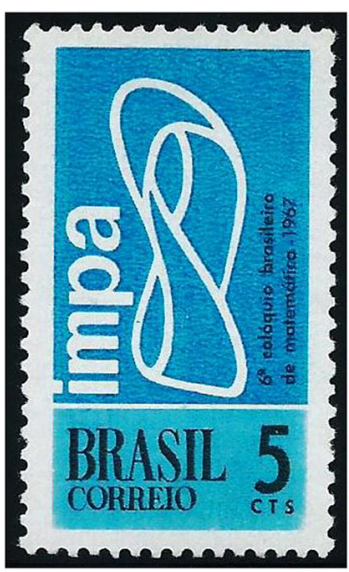

Möbius band

> Column editor's address: Robin Wilson, Mathematical Institute,

Andrew Wiles Building, University of Oxford, UK

e-mail: r.j.wilson@open.ac.uk 